\title{
Voltage Mode Universal Filter Using Current Differencing Buffered Amplifier as an Active Device
}

\author{
Sheikh Ajaz Bashir*, Nisar Ahmed Shah \\ Department of Electronics \& Instrumentation Technology, University of Kashmir, Srinagar, India \\ Email: *iamsheikhajaz@gmail.com
}

Received May 2, 2012; revised June 2, 2012; accepted June 9, 2012

\begin{abstract}
Integrated filter circuit design resulted in desire for replacement of Inductors by active elements like Operational Amplifiers which led to the introduction of active filters. Active filter design has evolved over a period of time. Starting with OP AMPs, we have witnessed phenomenal growth of active component usage in filter design and development catering to varying requirements. This has contributed greatly in emergence of circuits with minimal limitations and advantages in terms of wide Bandwidth and High slew rates. Current Differencing Buffered Amplifier (CDBA) based active filter design has resulted in introduction of many novel circuits. This paper proposes a new Voltage-mode three input and Single output (TISO) multifunction filter based on single CDBA, four resistors and two capacitors. This second order filter circuit is capable of realizing various filter functions by choosing values of the three inputs variably. The natural frequency $\left(\omega_{0}\right)$ can be tuned with passive components and the $\mathrm{Q}$ of the circuit is independent of $\left(\omega_{0}\right)$. The higher cascading capability of the circuit is ensured because of its low-output impedance. Further PSPICE-simulated results are in conformity with theoretical values.
\end{abstract}

Keywords: CDBA; Voltage Mode Circuits; Multifunction Filters; Slew Rate; Parasitic Capacitance

\section{Introduction}

CDBA, a current-mode component, has been introduced by C. Acar and S. Ozoguz in 1999 [1]. It offers advantageous features such as high slew rate, absence of parasitic capacitance, wide bandwidth, and simple implementation. Since the CDBA consists of a unity-gain current differential amplifier and a unity-gain voltage amplifier, this element would be suitable for the implementation of voltage and current-mode signal processing applications. As far as the applications of the CDBA are concerned, various voltage-mode and current-mode filters and oscillators have been reported in literature [210]. CDBA being a Current-mode universal active component, provides wide bandwidth and high slew-rate as distinct advantages.

In this paper we are implementing CDBA, a current mode active element, in voltage mode operation due to the fact that majority of analog signal processing applications require voltage-mode operation [2].

The circuit symbol of the CDBA is shown in Figure 1(a), where $p$ and $n$ are the positive and negative current input terminals, respectively, $z$ is the current output terminal, and $w$ is the voltage output terminal. Its current and voltage characteristics can be described by the ma-

"Corresponding author. trix shown below.

$$
\left[\begin{array}{l}
I_{z} \\
V_{w} \\
V_{p} \\
V_{n}
\end{array}\right]=\left[\begin{array}{cccc}
0 & 0 & 1 & -1 \\
1 & 0 & 0 & 0 \\
0 & 0 & 0 & 0 \\
0 & 0 & 0 & 0
\end{array}\right]\left[\begin{array}{c}
V_{z} \\
I_{w} \\
I_{p} \\
I_{n}
\end{array}\right]
$$

Here we have

$$
V_{p}=V_{n}, I_{z}=I_{p}-I_{n} \text { and } V_{w}=V_{z}
$$

According to the above set of describing equations at (1) depicted by the Matrix, the terminal $z$ behaves as a current source that takes the difference of currents at the inputs, and the terminal $w$ behaves as a voltage source that copies the output voltage at the $z$ terminal. Thus, the CDBA can be considered as a collection of a non inverting and an inverting current-mode, and non-inverting voltage-mode unity-gain cells, which can be realized by a cascade connection of a current subtractor and a voltage follower. Although the CDBA can be realized by using several well-known circuit techniques, one possible practical implementation is given in Figure 1(b) by use of two CFAs (AD844) [11]. Further a CMOS circuit realization of the CDBA is displayed in Figure 2.

The size of CDBA configuration shown in Figure 2 is about the same as that of a CMOS II-generation Current 


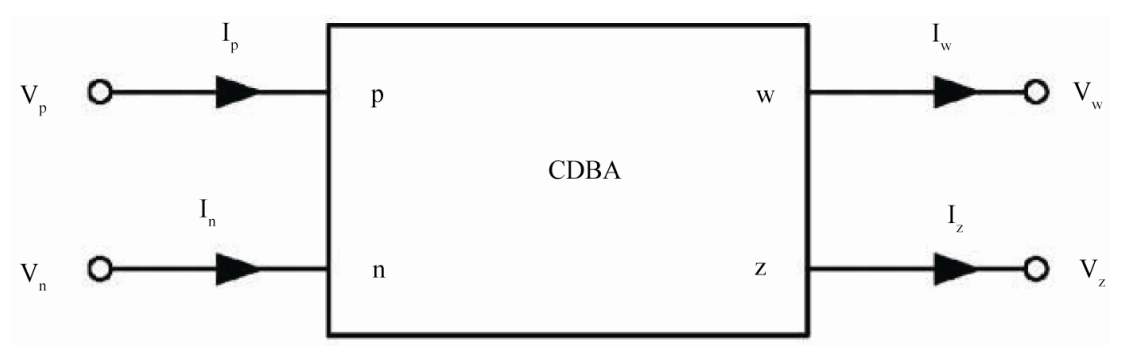

(a)

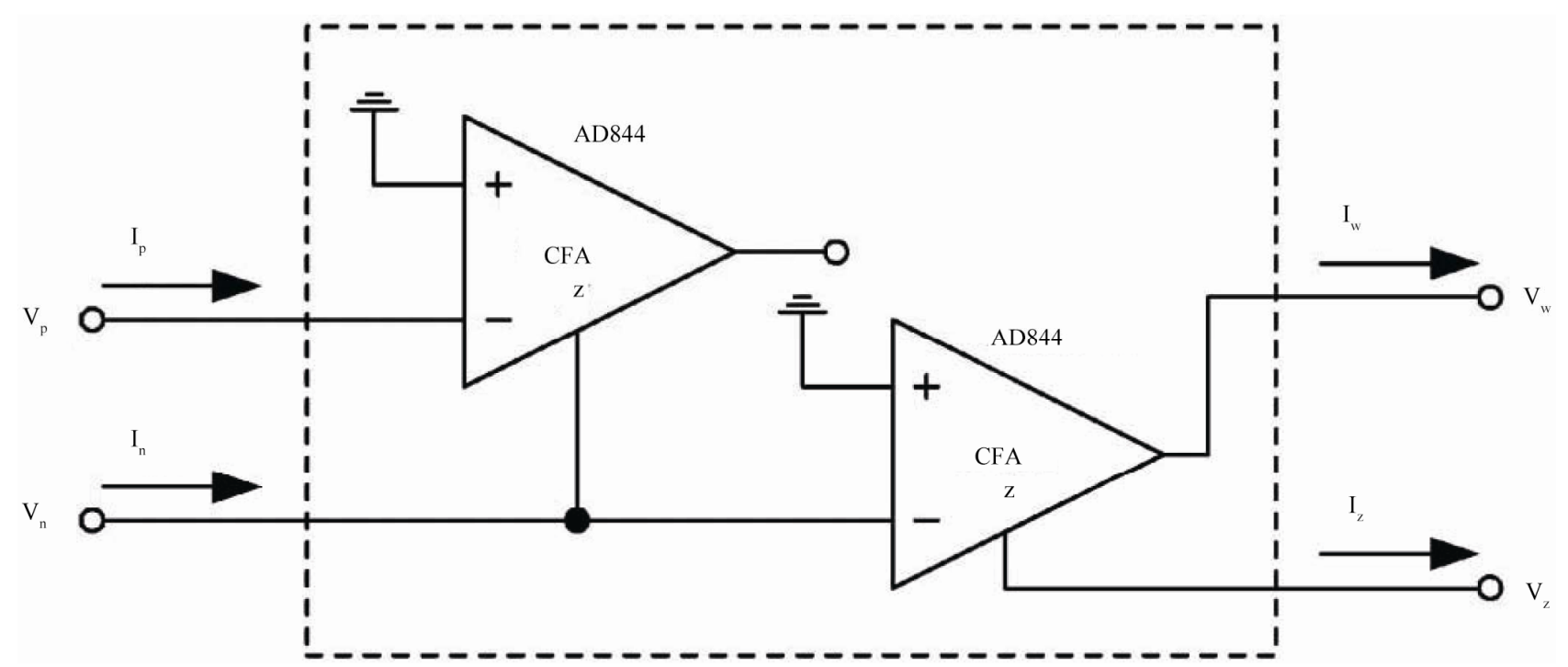

(b)

Figure 1. (a) CDBA symbol; (b) Implementation of CDBA using CFAs AD844.

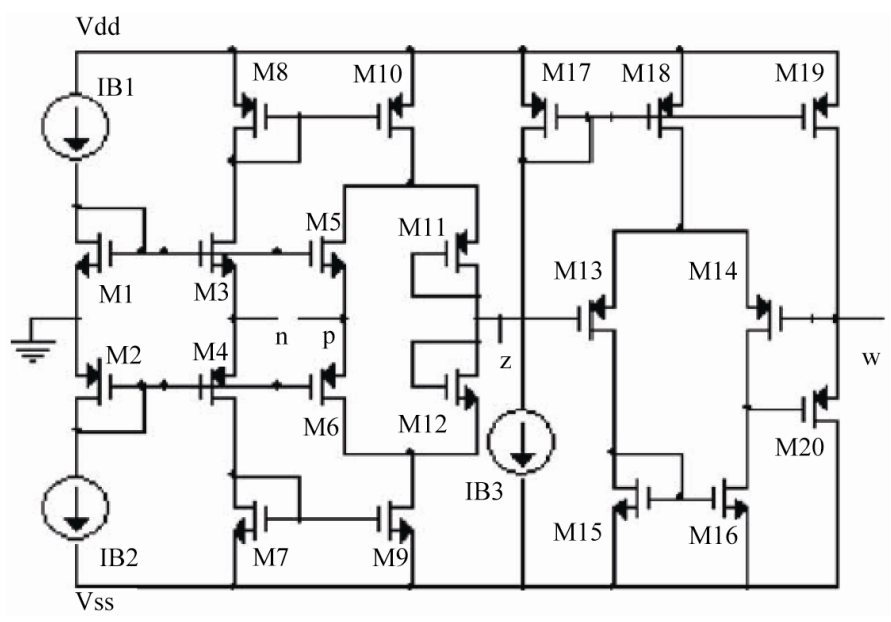

Figure 2. CMOS circuit realization of CDBA [11].

Conveyor (CC II) [12].

\section{Purpose of Research}

The aim of this research article is to present a Universal active second order filter with single active device and a few passive components and better cascadability. By suitable selection of inputs we should be able to realize all filter functions. The natural frequency of Biquad has to be dependent on passive components only with Quality factor being independent of it.

\section{Proposed Circuit}

In this paper a Voltage Mode multifunction circuit based on single CDBA is proposed as shown in Figure 3. In 
the proposed circuit a single CDBA, four resistors and two capacitors are used and the circuit shapes as a three input and single Output unit (TISO).

From routine Circuit analysis, the characteristic equation of the proposed CDBA based VM TISO circuit can be written as at (2).

$$
V_{\text {out }}=\frac{V_{1} s^{2}+V_{2} s \frac{1}{R_{4} C_{1}}+V_{3} \frac{1}{R_{3} R_{5} C_{1} C_{6}}}{s^{2}+\frac{s}{C_{1}}\left(\frac{1}{R_{3}}+\frac{1}{R_{4}}+\frac{1}{R_{5}}\right)+\frac{1}{R_{2} R_{3} C_{1} C_{6}}}
$$

The natural frequency of the circuit $\left(\omega_{0}\right)$ will be as shown in Equation (3). Here the value of $\left(\omega_{0}\right)$ can be adjusted by changing the values of passive components $R_{2}$ and $R_{3}$.

$$
\omega_{o}=\sqrt{\frac{1}{R_{2} R_{3} C_{1} C_{6}}}
$$

Further the Quality factor of the filter block will be derived from Equation (4) where its value will be independent of $\left(\omega_{0}\right)$ and can be adjusted with variation in $R_{4}$ only.

$$
Q=\frac{R_{4}}{R_{2} R_{3}+R_{4} R_{3}+R_{2} R_{4}} \sqrt{\frac{R_{2} R_{3} C_{1}}{C_{6}}}
$$

\begin{tabular}{|c|c|c|}
\hline \multicolumn{2}{|c|}{ INPUT VALUES } & FILTER REALIZATION \\
\hline$V_{2}=V_{3}=0$ & $V_{1}=V_{\text {in }}$ & ad Order High Pass Filter \\
\hline$V_{2}=V_{3}=0$ & $V_{2}=V_{\text {in }}$ & A Second Order Band Pass Filter \\
\hline$V_{1}=V_{2}=0$ & $V_{3}=V_{\text {in }}$ & Filter \\
\hline$V_{2}=0$ & $V_{3}=V_{\text {in }}$ & A Second Order Notch Filter \\
\hline$V_{1}=-V_{2}=$ & $=V_{\text {in }}$ & A Second Order All Pass Filter \\
\hline
\end{tabular}

Realization of various filter topologies is possible by varying the inputs as under

\section{Circuit Simulation}

The performances of the proposed circuit given in Figure 3 has been simulated with PSPICE program. In the simulations, the CDBA was constructed employing commercially available current feedback amplifiers (CFAs), i.e., AD844 of Analog Devices, as given in Figure 1(b). The circuit was supplied with symmetrical voltages of $\pm 12 \mathrm{~V}$.

The simulated frequency characteristics of all the filter functions of the proposed CDBA-based VM multifunction TISO circuit in Figure 3 are shown in Figure 4 with the passive component values: $R_{2}=R_{3}=R_{4}=R_{5}=1 \mathrm{~K}$ Ohm, $C_{1}=C_{6}=1 \mathrm{nF}$ leading to $f_{\mathrm{o}}=159.15 \mathrm{KHz}$ and $Q=$ $1 / 3$ (Gain for BP response at $f_{\mathrm{o}}=0.3$ ). The filter is de-

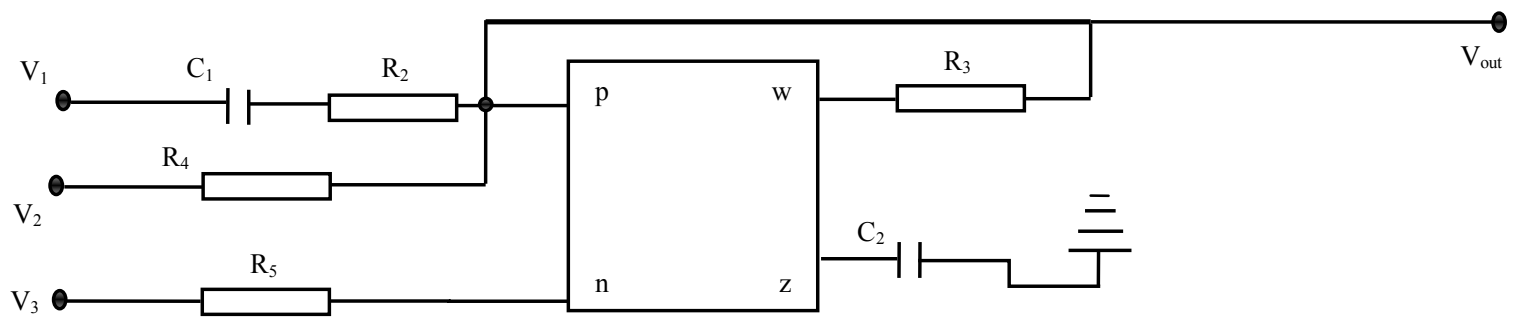

Figure 3. Proposed VM TISO multifunction filter.

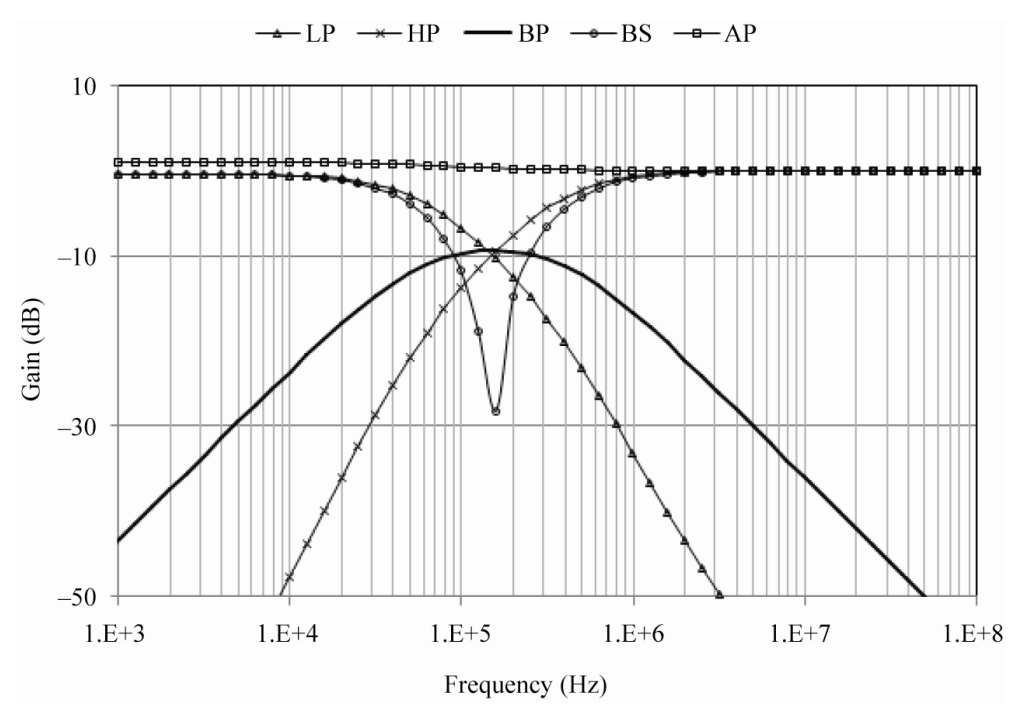

Figure 4. Magnitude response of proposed CDBA based voltage mode multifunction filter with $R_{2}=R_{3}=R_{4}=R_{5}=1 \mathrm{Kohm}$, $C_{1}=C_{6}=1 \mathrm{nF}$ leading to $f_{0}=159.15 \mathrm{KHz}$ and $Q=1 / 3$ (Gain for $\mathrm{BP}$ response at $f_{0}=0.3$ ). 


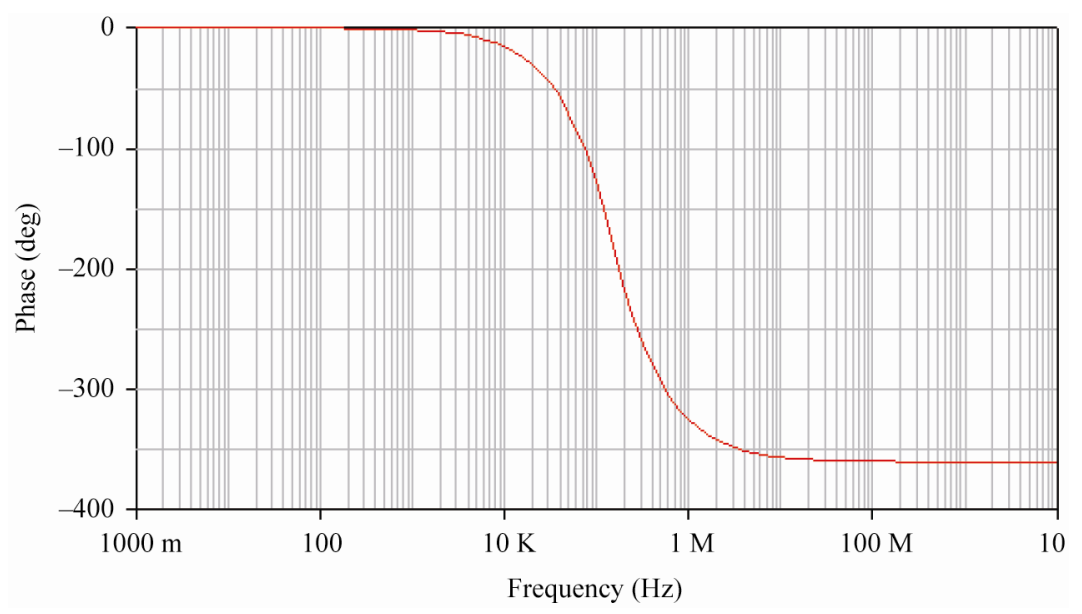

Figure 5. Phase response of all pass response.

signed for a natural angular frequency of $f_{o}=\omega_{0} / 2 \pi \cong$ $159.15 \mathrm{KHz}$ and the quality factor of $Q=1 / 3$. With the same setting, the simulated gain and phase responses of the AP filters verifying theory values are depicted in Figure 5. From the figures, it appears that the simulation results are in excellent agreement with theoretical values.

\section{Conclusion}

In this paper, VM TISO multifunction filter involving a single CDBA is introduced. The proposed circuit is able to realize Low Pass, High Pass, Band Pass, All Pass and Notch function as well. Besides employing a single CDBA, there are four resistive and two reactive components in the circuit block. Further incase of proposed circuit $Q$ is independent of $\left(\omega_{0}\right)$ and can be varied by simply changing the value of $R_{4}$ only. The tuning of filter i.e; changing the value of $\left(\omega_{0}\right)$ is possible by changing the value of passive components $R_{2}$ and $R_{3}$ in the circuit.

\section{REFERENCES}

[1] C. Acar and S. O" Zoǧuz, "A New Versatile Building Block: Current Differencing Buffered Amplifier Suitable for Analog Signal Processing Filters," Microelectronics Journal, Vol. 30, No. 2, 1999, pp. 157-160. doi:10.1016/S0026-2692(98)00102-5

[2] A. U. Keskin, "Voltage-Mode High-Q Band-Pass Filters and Oscillators Employing Single CDBA and Minimum Number of Components," International Journal of Electronics, Vol. 92, No. 8, 2005, pp. 479-487. doi:10.1080/00207210500168626

[3] S. O"zog^uz, A. Toker and C. Acar, "Current-Mode Continuous Time Fully-Integrated Universal Filter Using CDBAs," Electronics Letters, Vol. 35, No. 2, 1999, pp. 97-98. doi:10.1049/el:19990118
[4] W. Tangsrirat and W. Surakampontorn, "Realization of Multiple-Output Biquadratic Filters Using Current Differencing Buffered Amplifiers," International Journal of Electronics, Vol. 92, No. 6, 2005, pp. 313-325. doi: $10.1080 / 00207210500141862$

[5] W. Tangsrirat, "Voltage-Mode Multifunction Biquadratic Filter and Sinusoidal Oscillator Using Only Two CDBAs," Journal of Active and Passive Electronic Devices, Vol. 4, No. 4, 2009, pp. 335-345.

[6] H. Sedef and C. Acar, "On the Realization of VoltageMode Filters Using CDBA," Frequenz, Vol. 54, No. 9-10, 2000, pp. 198-202. doi:10.1515/FREQ.2000.54.9-10.198

[7] K. N. Salama and A. M. Soliman, "Voltage Mode Kerwin-Huelsman-Newcomb Circuit Using CDBAs," Frequenz, Vol. 54, No. 7-8, 2000, pp. 90-93.

[8] S. Maheshwari and I. A. Khan, "Novel Voltage-Mode Universal Filter Using Only Two CDBAs," Journal of Circuits, Systems and Computers, Vol. 14, No. 1, 2005, pp. 159-164. doi: $10.1142 / \mathrm{S} 0218126605002118$

[9] S. Ozoguz, A. Toker and C. Acar, "Current-Mode Continuous-Time Fully Integrated Universal Filter Using CDBAs," Electronics Letters, Vol. 35, No. 2, 1999, pp. 97-98. doi:10.1049/el:19990118

[10] A. U. Keskin and E. Hancioglu, "Current Mode Multifunction Filter Using Two CDBAs," International Journal of Electronics \& Communications, Vol. 59, No. 8, 2005, pp. 495-498.

[11] Anonymity, "Analog Devices Linear Products Data Book," Analog Devices, Norwood, 1990.

[12] W. S. Hassanein, I. A. Awad and A. M. Soliman, "New Wide Band Low Power CMOS Current Conveyors," Analog Integrated Circuits Signal Process, Vol. 40, No. 1, 2004, pp. 91-97. doi:10.1023/B:ALOG.0000031439.62496.f9 